\title{
Association of pleural effusion with an early molecular response in patients with newly diagnosed chronic-phase chronic myeloid leukemia receiving dasatinib: Results of a D-First study
}

\author{
MAKI HAGIHARA $^{1}$, NORIYOSHI IRIYAMA ${ }^{2}$, CHIKASHI YOSHIDA ${ }^{3}$, HISASHI WAKITA $^{4}$, SHIGERU CHIBA ${ }^{5}$, \\ SHINICHIRO OKAMOTO ${ }^{6}$, KIMIHIRO KAWAKAMI $^{7}$, NAOKI TAKEZAKO $^{8}$, TAKASHI KUMAGAI ${ }^{9}$, \\ KOITI INOKUCHI ${ }^{10}$, KAZUMA OHYASHIKI ${ }^{11}$, JUN TAGUCHI ${ }^{12}$, SHINGO YANO ${ }^{13}$, TADAHIKO IGARASHI ${ }^{14}$, \\ YASUJI KOUZAI ${ }^{15}$, SATOSHI MORITA ${ }^{16}$, JUNICHI SAKAMOTO ${ }^{17}$ and HISASHI SAKAMAKI ${ }^{18}$
}

\begin{abstract}
${ }^{1}$ Department of Hematology, Yokohama City University Medical Center, Kanagawa; ${ }^{2}$ Division of Hematology and Rheumatology, Department of Medicine, Nihon University School of Medicine, Tokyo; ${ }^{3}$ Department of Hematology, National Hospital Organization Mito Medical Center, Ibaraki; ${ }^{4}$ Division of Hematology and Oncology, Japanese Red Cross Society, Narita Red Cross Hospital, Chiba; ${ }^{5}$ Department of Hematology, Faculty of Medicine, University of Tsukuba, Ibaraki; ${ }^{6}$ Division of Hematology, Department of Internal Medicine, Keio University School of Medicine, Tokyo; ${ }^{7}$ Department of Hematology and Clinical Oncology, Kagawa Prefectural Central Hospital, Kagawa; ${ }^{8}$ Department of Hematology, National Hospital Organization Disaster Medical Center, Tokyo;

${ }^{9}$ Department of Hematology, Ohme Municipal General Hospital, Tokyo; ${ }^{10}$ Division of Hematology, Department of Internal Medicine, Nippon Medical School, Tokyo; ${ }^{11}$ Department of Hematology, Tokyo Medical University, Tokyo; ${ }^{12}$ Department of Hematology, Japanese Red Cross Shizuoka Hospital, Shizuoka;

${ }^{13}$ Division of Clinical Oncology and Hematology, Department of Internal Medicine, Jikei University School of Medicine, Tokyo; ${ }^{14}$ Division of Hematology and Oncology, Gunma Prefectural Cancer Center, Gunma; ${ }^{15}$ Department of Hematology, Tokyo Metropolitan Tama Synthesis Medical Center, Tokyo; ${ }^{16}$ Department of Biomedical Statistics and Bioinformatics, Kyoto University Graduate School of Medicine, Kyoto; ${ }^{17}$ Tokai Central Hospital, Gifu;

${ }^{18}$ Hematology Division, Tokyo Metropolitan Cancer and Infectious Diseases Center, Komagome Hospital, Tokyo, Japan
\end{abstract}

Received April 22,2016; Accepted September 7, 2016

DOI: $10.3892 / o r .2016 .5110$

\begin{abstract}
Despite the efficacy and safety of dasatinib treatment for chronic-phase chronic myeloid leukemia (CML-CP), adverse effects such as pleural effusion (PE) are still a serious concern. We determined the clinical significance of PE incidence using patient data derived from the D-First clinical study. In the present study, chest radiography and quantification of specific lymphocyte subsets were performed routinely after initiation of dasatinib treatment. Among 52 patients with newly diagnosed CML-CP, 17 (33\%) developed PE within 18 months after initial dasatinib administration, but all cases were moderate (Grade 1, 10 patients; Grade 2, 7 patients).
\end{abstract}

\footnotetext{
Correspondence to: Dr Noriyoshi Iriyama, Division of Hematology and Rheumatology, Department of Medicine, Nihon University School of Medicine, Tokyo, Japan

E-mail: iriyama.noriyoshi@nihon-u.ac.jp
}

Key words: chronic myeloid leukemia, newly-diagnosed, dasatinib, pleural effusion, molecular response
CD56 $6^{+}$lymphocyte counts at 1 month correlated significantly with the incidence of PE, whereas lymphocytosis did not. The major molecular response (MMR) rate at 3 months (although not at later times) was significantly higher in PE-positive patients than PE-negative patients (59\% versus $24 \%$, respectively; $\mathrm{P}=0.013$ ). Deep molecular response rates did not differ significantly between the PE groups at any time point during the observation period. Our results suggest that an immunemediated mechanism involving natural killer cells underlies the development of PE in patients receiving dasatinib for 18 months. This mechanism likely promotes transient tumor regression in patients newly diagnosed with CML-CP.

\section{Introduction}

Use of the tyrosine kinase inhibitor (TKI) imatinib has dramatically improved the outcome of patients with chronic myeloid leukemia (CML). The second-generation TKIs, nilotinib and dasatinib, are treatment options for patients with chronic-phase CML (CML-CP) who are intolerant or resistant to imatinib (1). Of serious concern, however, are the adverse effects associated with TKI treatment (2). Such effects include pleural effusion (PE) in dasatinib-treated patients, which is clinically important because it often curtails adherence to 
treatment regimens. On the other hand, PE is associated with lymphocytosis and favorable outcomes and thus is likely induced by an immune-mediated mechanism (3-5).

Dasatinib is currently available as a first-line treatment for newly diagnosed CML-CP. However, except the DASISION study, all previous studies examining dasatinib-associated PE were conducted in patients receiving dasatinib as a secondline therapy. The DASISION study, which compared the efficacy and safety of dasatinib and imatinib $(6,7)$, found that PE was infrequent and moderate when dasatinib was used as the first-line treatment rather than the second-line treatment (6). Whether PE affects the treatment response or acts via an immunological mechanism is not known. Comprehensive evaluation of the clinical significance of PE in first-line dasatinib treatment is needed, and toward this goal, we analyzed data derived from patients enrolled in the D-First study, a clinical trial of CML-CP patients receiving first-line dasatinib therapy. Specifically, we identified the risk factors for PE and the prognostic implications of PE.

\section{Patients and methods}

Patients. The open-label, multicenter, prospective 'D-First' trial (ClinicalTrials.gov, NCT01464411) was used to derive data for the current study (8). It excluded CML-CP patients who were pregnant or breast feeding or had comorbidities that rendered them unsuitable for dasatinib therapy (e.g., a corrected QT interval $>450 \mathrm{msec}$ on an electrocardiogram, double cancer, PE, or history of severe or recent cardiovascular disease). Fifty-two patients with newly diagnosed CML-CP were enrolled and received $100 \mathrm{mg}$ of dasatinib once daily. One of the 52 patients did not express the $B C R-A B L 1$ transcript and was included in the PE incidence analysis, but not the molecular response analysis. The study was approved by the research ethics boards of all participating institutions and was conducted in accordance with the Declaration of Helsinki. All patients gave written informed consent prior to enrollment.

Assessment of the treatment response and molecular analysis. $B C R-A B L 1$ mRNA expression was used to assess the molecular response to dasatinib. Peripheral blood samples were drawn from patients 3 months after initiating dasatinib treatment, and amounts of the $B C R-A B L 1$ transcript were quantified by Biomedical Laboratories (BML; Tokyo, Japan) via real-time quantitative polymerase chain reaction. The data were normalized to the housekeeping gene $G A P D H$ and converted to the International Scale $\left(B C R-A B L 1^{I S}\right)(9,10)$. A major molecular response (MMR) and a deep molecular response (DMR) were defined as $<0.1 \%$ and $<0.0069 \%$ of $B C R-A B L I^{I S}$, respectively (11).

Assessment of PE. PE was evaluated by chest radiographs, which were routinely performed at 2 weeks and at 1, 2, 3, 6, $9,12,15$, and 18 months after the initiation of dasatinib treatment. Patients were excused from chest radiography at the physician's discretion if symptoms or abnormal findings were not observed. Toxicities were graded according to the National Cancer Institute Common Terminology Criteria for Adverse Events, version 4.0.

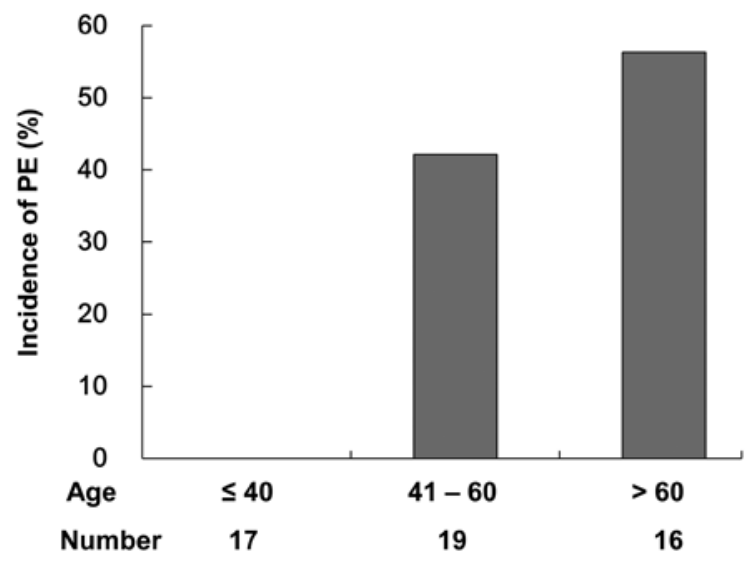

Figure 1. Cumulative incidence rates of pleural effusion (PE) after commencing dasatinib treatment stratified by patient age. The differences between the groups were significant, with the highest incidence rate in the oldest group $(\mathrm{P}<0.01)$.

Flow cytometric analysis and definition of lymphocytosis. Immunophenotyping of lymphocyte fractions in peripheral blood samples was performed at BML using standard immunofluorescence methods and anti-human antibodies recognizing the CD3, CD8, CD56, and CD57 surface antigens. Antigen expression was evaluated using a FACSCalibur ${ }^{\mathrm{TM}}$ system (Becton-Dickinson; NJ, USA). Data were collected using CELLQuest, version 3.3 (Becton-Dickinson) and analyzed using original software from BML. Each lymphocyte subset was quantified as follows: total leukocyte count $\mathrm{x}$ percentage of lymphocytes $\mathrm{x}$ percentage of each lymphocyte subset. Lymphocytosis was defined as an absolute blood lymphocyte count $>3.6 \times 10^{3} / \mu 1$ on $\geq 2$ occasions after $\geq 4$ weeks of therapy. Blood lymphocyte analyses were performed monthly after initiating treatment.

Statistical analyses. The Fisher's exact test and Mann-Whitney $\mathrm{U}$ test were used to determine statistical significance. Receiver operating characteristic (ROC) curves were generated to evaluate the correlation between PE and specific leukocyte subsets, and optimal thresholds along the ROC curves were determined using the Youden index. Patients who discontinued dasatinib treatment for any reason before achieving an MMR or a DMR were considered to have no MMR or DMR at 18 months. A P-value $<0.05$ was considered significant; a P-value $<0.1$ indicated a tendency toward significance. All statistical analyses were performed using EZR (Saitama Medical Center, Jichi Medical University), the graphical user interface supplied by the R Foundation for Statistical Computing.

\section{Results}

Results of treatment in the study population. No mortality or disease progression occurred within the first 18 months of dasatinib treatment. Three of the 52 enrolled patients discontinued treatment prior to 18 months (one at 1 month and two at 11 months) because of intolerance to dasatinib. Regarding molecular assessment, 38 of 51 patients (75\%; 95\% confidence interval [CI], 60-86\%) had an MMR within 12 months after treatment initiation, and $30(59 \% ; 95 \% \mathrm{CI}, 44-72 \%)$ had a DMR within 18 months. 

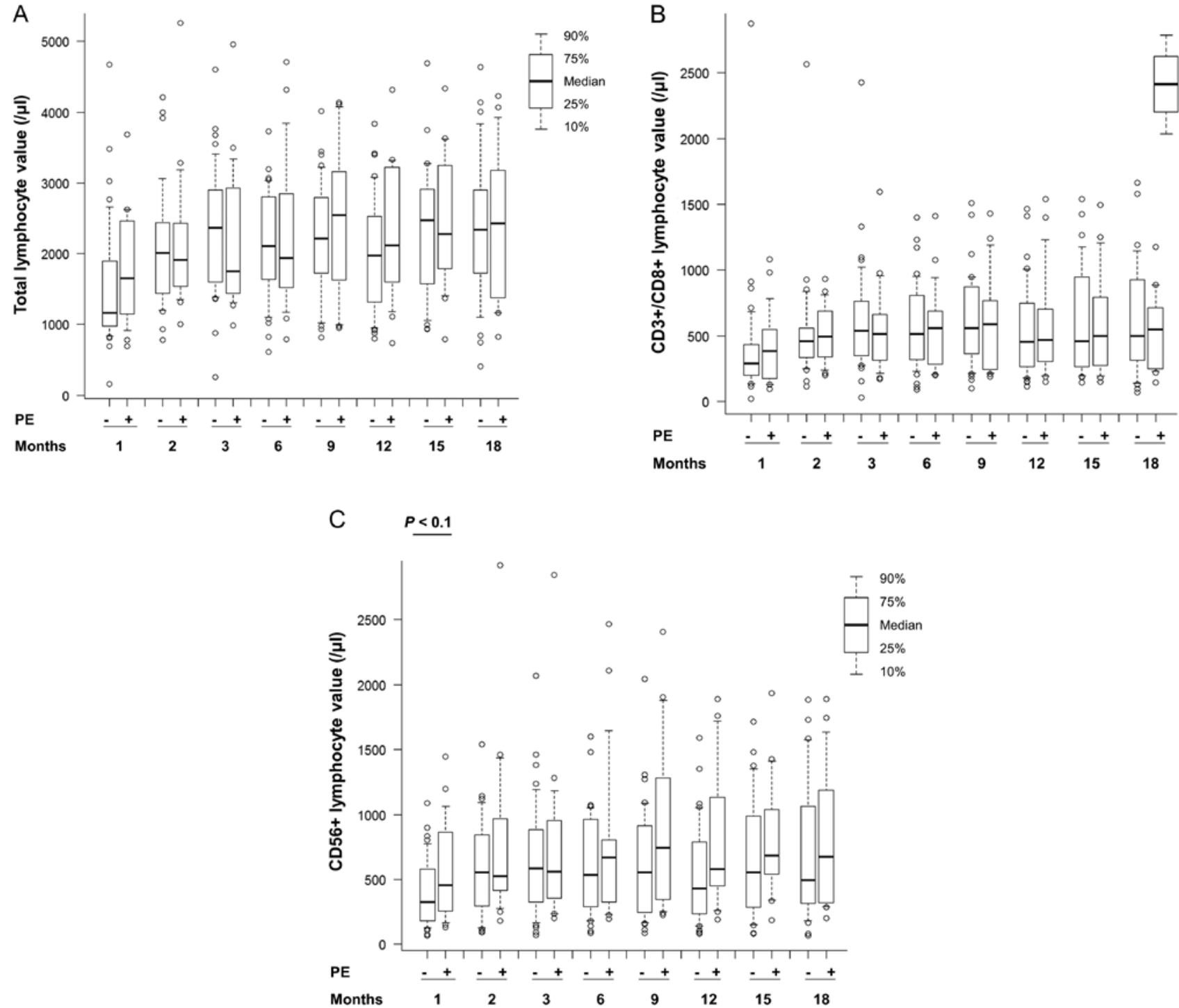

Figure 2. Box-and-whisker plots showing lymphocyte counts $/ \mu 1$ in the presence (+) or absence (-) of pleural effusion (PE) at 18 months. (A) Total lymphocytes. (B) $\mathrm{CD}^{+} / \mathrm{CD} 8^{+}$lymphocytes. (C) $\mathrm{CD} 56^{+}$lymphocytes. Although there were no significant differences plus/minus PE, the values for $\mathrm{CD} 56^{+}$ lymphocytes at 1 month tended to differ $(\mathrm{P}<0.1)$. The I bar, box, and black line indicate $90 \%$ (upper) and 10\% (lower), $75 \%$ (upper) and $25 \%$ (lower), and median values, respectively.

Cumulative incidence of PE and patient characteristics. The overall incidence rate of PE 18 months after treatment initiation was $33 \%$, and the median time to development of PE was 9 months (1-15 months). At 6 and 12 months, the cumulative incidence rates of PE were 14 and $27 \%$, respectively. Among the 17 patients who developed PE, 10 (59\%) had grade $1 \mathrm{PE}$ and $7(41 \%)$ had grade $2 \mathrm{PE}$. The characteristics of patients with and without PE at 18 months are shown in Table I. Although unrelated to gender, body weight, Sokal score, and $B C R-A B L 1$ transcript expression, the incidence of PE significantly correlated with age. When patients were divided into three age groups, the PE incidence rate differed significantly between the groups and was highest in the oldest group ( $>60$ years, $\mathrm{P}<0.01$ ) (Fig. 1).

Association of PE incidence and development of lymphocytosis. We identified 14 patients (27\%) who developed lymphocytosis during the observation period. The incidence rates of $\mathrm{PE}$ in patients with and without lymphocytosis were $30.4 \%$ and $34.5 \%$, respectively, and did not differ significantly.

Lymphocyte dynamics according to the incidence of PE. To examine lymphocyte dynamics, $\mathrm{CD} 56^{+}$lymphocytes [natural killer (NK) cells], and $\mathrm{CD}^{+} / 8^{+}$lymphocytes [cytotoxic T-lymphocytes (CTLs)] were separated from whole lymphocyte fractions at each time point (Fig. 2A-C). There were no significant differences in the levels of unfractionated, CD $56^{+}$, or $\mathrm{CD}^{+} / 8^{+}$lymphocytes between patients who developed PE and those who did not. However, in the first month following dasatinib treatment, the $\mathrm{CD} 56^{+}$fraction tended to be larger in PE-positive patients than PE-negative patients $(\mathrm{P}<0.1)$.

To better evaluate the association between the incidence of $\mathrm{PE}$ and the level of CD56+ lymphocytes 1 month after dasatinib treatment, we generated ROC curves and calculated the threshold for CD56 lymphocytes (Fig. 3A). Using this threshold, we divided the patients into two groups: those with high $(\geq 428 / \mu 1)$ and low $(<428 / \mu 1)$ CD56 ${ }^{+}$lymphocyte levels. 
Table I. Baseline characteristics of patients with or without pleural effusion (PE) by 18 months.

\begin{tabular}{|c|c|c|c|}
\hline Factors & $\mathrm{PE}^{+}(\mathrm{N}=17)$ & $\mathrm{PE}^{-}(\mathrm{N}=35)$ & P-value \\
\hline Age (years), median (range) & $63(47-81)$ & $41(23-82)$ & 0.0013 \\
\hline Gender (male/female) & $9 / 8$ & $22 / 13$ & 0.56 \\
\hline Body weight (kg) & $59(35-87)$ & $63.5(45-88)$ & 0.62 \\
\hline Sokal score & & & 0.24 \\
\hline Low-risk & 5 & 19 & \\
\hline Intermediate-risk & 10 & 12 & \\
\hline High-risk & 2 & 4 & \\
\hline $\begin{array}{l}B C R-A B L 1 \text { transcript value } \\
\text { (copies/ } \mu \mathrm{g} \text { RNA), median (range) }\end{array}$ & $220,000(64,000-700,000)$ & $265,000(71,000-1,000,000)$ & 0.27 \\
\hline
\end{tabular}
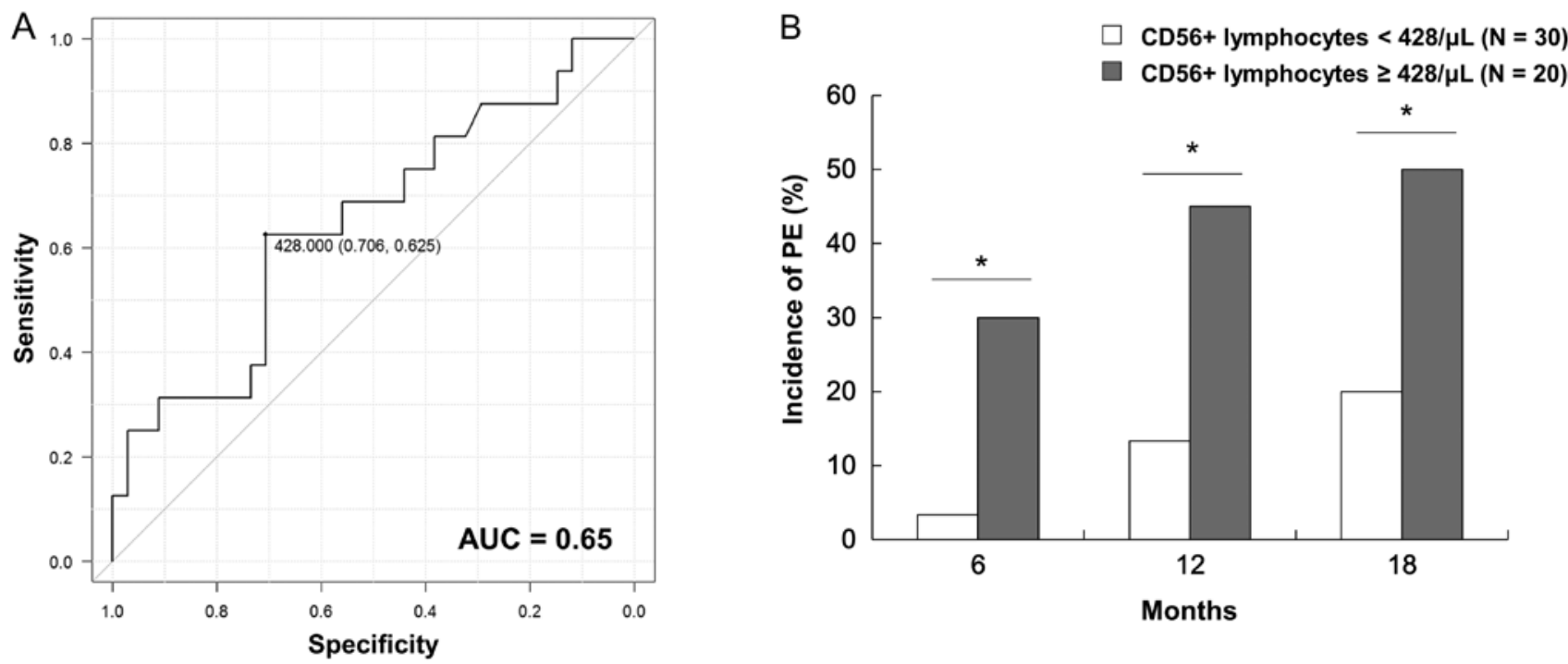

Figure 3. A receiver operating characteristic (ROC) curve showing the association between the incidence of pleural effusion (PE) at 18 months and the values for CD56 $6^{+}$lymphocytes at 1 month (A). The ROC curve indicates a correlation [area under the curve (AUC), 0.65] with the highest Youden index at 428/ $\mu$ l CD56 $6^{+}$ lymphocytes. (B) The cumulative incidence of PE stratified by CD56 lymphocyte values is shown. The cumulative incidence rate of PE was significantly higher in the $\geq 428 / \mu 1$ group than the $<428 / \mu 1$ group throughout the observation period. ${ }^{*} \mathrm{P}<0.05$.

The incidence of PE was significantly higher in the former (Fig. 3B), and this difference was maintained up to 18 months. To determine whether a specific $\mathrm{CD}^{+} 6^{+}$subset accounted for this increase, we counted $\mathrm{CD} 56^{+}$lymphocytes separated according to CD3 and CD57 status 1 month after initial dasatinib treatment (Fig. 4). Although the association between the incidence of PE and CD3 or CD57 status was not significant, $\mathrm{CD} 6^{+} / \mathrm{CD} 3^{-}$and $\mathrm{CD} 56^{+} / \mathrm{CD} 57^{+}$levels tended to be higher in PE-positive patients than PE-negative patients (Fig. 4).

Patient response rate according to the incidence of $P E$. To investigate the relationship between PE incidence and treatment response, we determined the cumulative MMR and DMR rates at 3, 6, 9, 12, 15, and 18 months after the initiation of dasatinib treatment in PE-positive and PE-negative patients. At all time points, cumulative MMR rates were higher in the PE-positive group. Importantly, the differences were only significant at 3 months, after which they narrowed until the rate in both groups was nearly equal at 18 months (Fig. 5A). DMR rates also tended to be higher in the PE-positive group at all time points, but not significantly so (Fig. 5B).

\section{Discussion}

Currently, dasatinib is used not only as a second-line treatment for relapsed or refractory CML, but also as a first-line treatment, for which it was very efficacious in a large-scale randomized trial (11). In both first-line and second-line dasatinib treatments, the incidence of PE is considered to be a common toxicity (2), but the mechanism by which dasatinib causes PE and the impact of PE on treatment outcome are unclear. We therefore prospectively investigated the clinical characteristics of newly diagnosed CML-CP patients given dasatinib and closely analyzed the relationship between treatment outcome, PE occurrence, and the immunomodulatory effects of dasatinib. 


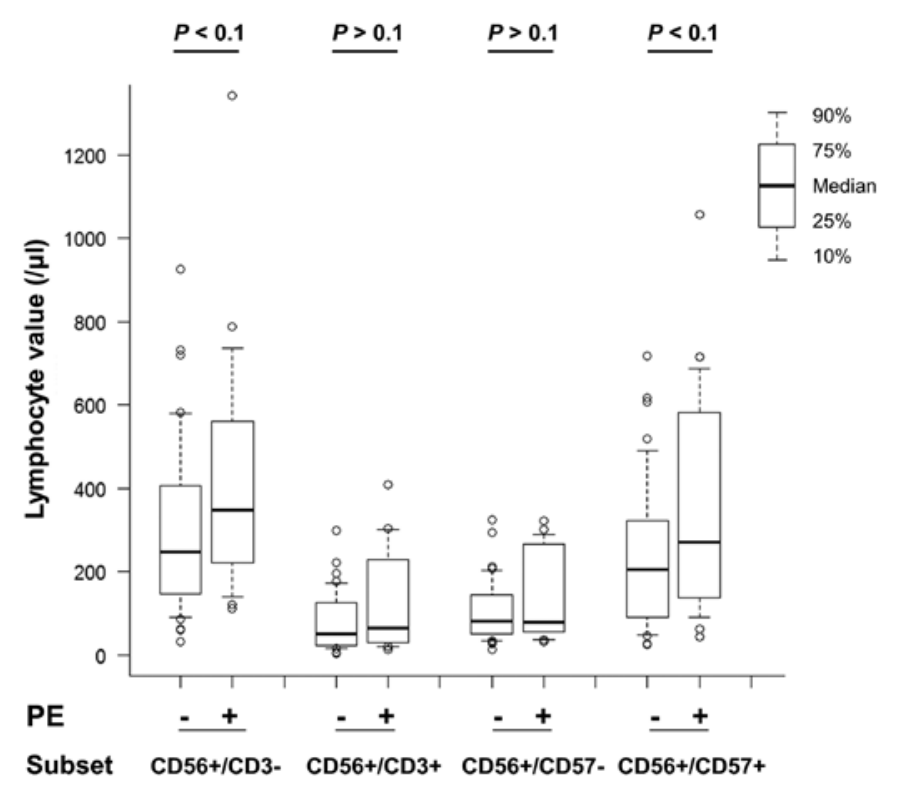

Figure 4. Values for the lymphocyte subsets at 1 month in the presence (+) or absence (-) of pleural effusion (PE). Although there were no significant differences plus/minus PE, the values for $\mathrm{CD} 56^{+} / \mathrm{CD}^{+}$and $\mathrm{CD} 56^{+} / \mathrm{CD} 57^{+}$ lymphocytes tended to be higher in the $\mathrm{PE}^{+}$group.

In the current study, the incidence of PE was associated with high molecular response rates at early times after the initiation of dasatinib treatment and with an immune response as indicated by NK cell counts at 1 month after initial treatment. Most published data on dasatinib-associated PE were generated in patients for whom dasatinib was the second-line therapy after imatinib (4,12-14). By comparing our results with those of previous studies of CML patients receiving dasatinib as a second-line therapy, we were able to assess the significance of PE prevalence in first-line therapy. Three points of interest in our study are discussed below.

First, PE was more frequent in older patients, similar to a previous study (15), but was unrelated to patient characteristics such as sex, body weight, and performance status. Previous studies analyzing the pharmacokinetics of dasatinib in secondline therapy showed that the risk of developing PE increased as patients aged (15-17). PE may occur more frequently in elderly patients because they may not metabolize dasatinib as efficiently as younger patients. Additional risk factors for PE in second-line dasatinib therapy include the steady state trough plasma concentration of dasatinib, cardiac disease, and hypertension $(15,18)$. However, patients with comorbidities were not eligible for our study, and therefore the association between cardiac disease and PE development was not analyzed.

Second, the occurrence of PE was related to the treatment response, especially the early molecular response. In patients with PE, the cumulative MMR rate was significantly higher in the PE-positive group than the PE-negative group only at 3 months after commencement of dasatinib treatment, while the DMR rate tended to be higher in the PE-positive group through the treatment period. The DMR results suggest that the mechanism underlying PE development likely promotes only transient tumor regression in the first-line setting.

Third, of particular interest in this study is the mechanism by which PE develops in response to dasatinib, particularly
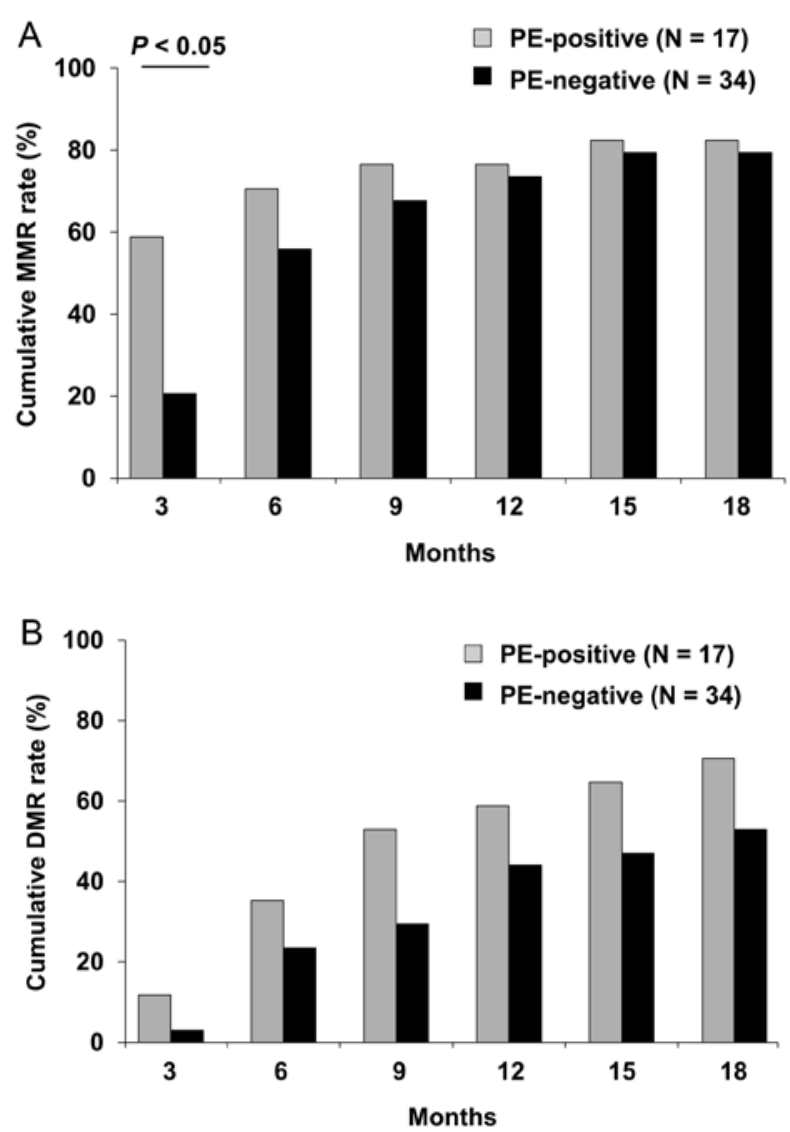

Figure 5. Cumulative major molecular response (MMR; A) and deep molecular response (DMR; B) rates in the presence $(+)$ and absence $(-)$ of pleural effusion (PE). MMR rates differed significantly between the PE groups at 3 months only. DMR rates did not differ significantly at any time point during the observation period.

in terms of the hypothetical immune-mediated pathway $(3,4)$. To date, many studies have reported an association between lymphocytosis, especially large granular lymphocytosis, and $\mathrm{PE}$ incidence in patients receiving dasatinib $(2,3,19)$. Although lymphocytosis did not correlate with $\mathrm{PE}$ incidence in our study, we demonstrated an expansion of CD56 ${ }^{+}$lymphocytes in patients with PE as early as 1 month after dasatinib treatment. We observed lower levels in CD56 ${ }^{+}$lymphocytes at 1 month compared to those at 2 or more months, suggesting that the proliferation of NK cells is inhibited at the time of diagnosis and increases after dasatinib therapy. In support, Kreutzman et al (20) reported the presence of monoclonal and oligoclonal NK cells and/or CTLs in newly diagnosed CML-CP patients. Cytotoxic cells presumably are present at low levels at the time of diagnosis but markedly increase in number after dasatinib administration, presumably to trigger immune surveillance against CML cells. A previous study assessing the significance of $\mathrm{CD}^{2} 6^{+} / \mathrm{CD} 57^{+}$pool size (21) showed that dasatinib-induced expansion of enhanced cytotoxic NK populations improved CML control. We suggest that an immune response involving NK cells occurring as early as 1 month after dasatinib administration increases the incidence of PE for up to 18 months and likely promotes rapid tumor regression within 3 months.

The incidence of PE in our study was $33 \%$, which is higher than that in the DASISION trial (14\% at 24 months) (7). Our 
study protocol may account in part for this discrepancy: it involved frequent chest X-rays to detect mild asymptomatic $\mathrm{PE}$, especially at early time points after initiation of dasatinib treatment. Alternatively or in addition, the different rates may reflect ethnic differences, which may influence dasatinib pharmacokinetics. In support, in a subset analysis, the DASISION trial detected a higher rate of PE in East Asian patients than non-East Asian patients $(22,23)$.

Although the incidence of PE was high in our study, the degree of PE was usually mild to moderate. The patients in our study did not require thoracentesis because PE was managed via dose reduction. Only two patients discontinued dasatinib treatment owing to uncontrolled grade $2 \mathrm{PE}$. Among the PE-positive patients who continued the core treatment, dasatinib doses were optimized for management of PE, with a mean daily dose of $75 \mathrm{mg}$ at 18 months. Most PE patients, however, received a mean daily dose of $90 \mathrm{mg}$ without ill effects. We did not investigate the management of PE other than dose optimization.

In summary, we prospectively examined dasatinib-related PE in newly diagnosed CML-CP patients, and we suggest that early expansion of NK populations may predict PE occurrence. Our results indicate that the incidence of $\mathrm{PE}$ up to 18 months after initial dasatinib treatment may be due to an immune-mediated mechanism involving NK cells. We suggest that this underlying mechanism likely contributes to transient tumor regression in patients newly diagnosed with CML-CP. This study not only reveals a possible mechanism of PE development, but also suggests that, similar to previous studies, the occurrence of PE may be related to favorable outcomes in patients with CML-CP.

\section{Acknowledgements}

This study was supported by the Epidemiological and Clinical Research Information Network (ECRIN). We thank Yumi Miyashita at ECRIN for collecting the data, and Yoshinori Yamamoto at BML for analyzing the data. N.I. received an honorarium and a speaker fee from Bristol-Myers Squibb and Novartis, respectively. T.K. received a lecture fee from Bristol-Myers Squibb. C.Y. received honoraria from Bristol-Myers Squibb and Novartis and grants from Otsuka Pharmaceutical. S.C. and K.O. received grants, speaker fees, and personal fees from Bristol-Myers Squibb and Novartis. S.C. has a pending patent for a diagnostic method for lymphoma. K.O. is a paid consult for Ariad. S.M. received a speaker fee from Bristol-Myers Squibb. S.O. received lecture fees and grant from Bristol-Myers Squibb.

\section{References}

1. Cortes J, Kim DW, Raffoux E, Martinelli G, Ritchie E, Roy L, Coutre S, Corm S, Hamerschlak N, Tang JL, et al: Efficacy and safety of dasatinib in imatinib-resistant or -intolerant patients with chronic myeloid leukemia in blast phase. Leukemia 22 2176-2183, 2008.

2. Quintás-Cardama A, Kantarjian H, O'brien S, Borthakur G, Bruzzi J, Munden R and Cortes J: Pleural effusion in patients with chronic myelogenous leukemia treated with dasatinib after imatinib failure. J Clin Oncol 25: 3908-3914, 2007.

3. Goldblatt M, Huggins JT, Doelken P, Gurung P and Sahn SA: Dasatinib-induced pleural effusions: A lymphatic network disorder? Am J Med Sci 338: 414-417, 2009.
4. Nagata Y, Ohashi K, Fukuda S, Kamata N, Akiyama H and Sakamaki H: Clinical features of dasatinib-induced large granular lymphocytosis and pleural effusion. Int J Hematol 91: 799-807, 2010.

5. Paydas S: Dasatinib, large granular lymphocytosis, and pleural effusion: Useful or adverse effect? Crit Rev Oncol Hematol 89: 242-247, 2014.

6. Laneuville P, Baccarani M, Cortes JE, Hochhaus A, Kantarjian H, Shah NP, Bradley-Garelik MB, Zhu C and Porkka K: Analysis of patients (pts) with chronic phase chronic myeloid leukemia (CML-CP) who develop pleural effusion on first-line dasatinib: Management and outcomes. J Clin Oncol 29 (Suppl): 6605, 2011.

7. Kantarjian HM, Shah NP, Cortes JE, Baccarani M, Agarwal MB, Undurraga MS, Wang J, Ipiña JJ, Kim DW, Ogura M, et al: Dasatinib or imatinib in newly diagnosed chronic-phase chronic myeloid leukemia: 2-year follow-up from a randomized phase 3 trial (DASISION). Blood 119: 1123-1129, 2012.

8. Iriyama N,Fujisawa S, Yoshida C, Wakita H, Chiba S, Okamoto S, Kawakami K, Takezako N, Kumagai T, Inokuchi K, et al: Shorter halving time of BCR-ABL1 transcripts is a novel predictor for achievement of molecular responses in newly diagnosed chronicphase chronic myeloid leukemia treated with dasatinib: Results of the D-first study of Kanto CML study group. Am J Hematol 90: 282-287, 2015.

9. Yoshida C, Fletcher L, Ohashi K, Wakita H, Kumagai T, Shiseki M, Matsuei K, Inokuchi K, Hatta Y, Shirasugi Y, et al: Harmonization of molecular monitoring of chronic myeloid leukemia therapy in Japan. Int J Clin Oncol 17: 584-589, 2012.

10. Kumagai T, Matsuki E, Inokuchi K, Ohashi K, Shinagawa A, Takeuchi J, Yoshida C, Okamoto S, Wakita H, Kozai Y, et al: Relative increase in lymphocytes from as early as 1 month predicts improved response to dasatinib in chronic-phase chronic myelogenous leukemia. Int J Hematol 99: 41-52, 2014.

11. Kantarjian H, Shah NP, Hochhaus A, Cortes J, Shah S, Ayala M, Moiraghi B, Shen Z, Mayer J, Pasquini R, et al: Dasatinib versus imatinib in newly diagnosed chronic-phase chronic myeloid leukemia. N Engl J Med 362: 2260-2270, 2010.

12. Kim D, Goh HG, Kim SH, Cho BS and Kim DW: Long-term pattern of pleural effusion from chronic myeloid leukemia patients in second-line dasatinib therapy. Int J Hematol 94: 361-371, 2011.

13. Latagliata R, Breccia M, Fava C, Stagno F, Tiribelli M, Luciano L, Gozzini A, Gugliotta G, Annunziata M, Cavazzini F, et al: Incidence, risk factors and management of pleural effusions during dasatinib treatment in unselected elderly patients with chronic myelogenous leukaemia. Hematol Oncol 31: 103-109, 2013.

14. Eskazan AE, Eyice D, Kurt EA, Elverdi T, Yalniz FF, Salihoglu A, Ar MC, Ongoren Aydin S, Baslar Z, Ferhanoglu B, et al: Chronic myeloid leukemia patients who develop grade I/II pleural effusion under second-line dasatinib have better responses and outcomes than patients without pleural effusion. Leuk Res 38 : 781-787, 2014.

15. Porkka K, Khoury HJ, Paquette RL, Matloub Y, Sinha R and Cortes JE: Dasatinib $100 \mathrm{mg}$ once daily minimizes the occurrence of pleural effusion in patients with chronic myeloid leukemia in chronic phase and efficacy is unaffected in patients who develop pleural effusion. Cancer 116: 377-386, 2010.

16. Wang X, Hochhaus A, Kantarjian HM, et al: Dasatinib pharmacokinetics and exposure-response (E-R): Relationship to safety and efficacy in patients (pts) with chronic myeloid leukemia (CML). J Clin Oncol 26 (Suppl): 3595, 2008.

17. Shah NP, Kantarjian HM, Kim DW, Réa D, Dorlhiac-Llacer PE, Milone JH, Vela-Ojeda J, Silver RT, Khoury HJ, Charbonnier A, et al: Intermittent target inhibition with dasatinib $100 \mathrm{mg}$ once daily preserves efficacy and improves tolerability in imatinibresistant and -intolerant chronic-phase chronic myeloid leukemia. J Clin Oncol 26: 3204-3212, 2008.

18. de Lavallade H, Punnialingam S, Milojkovic D, Bua M, Khorashad JS, Gabriel IH, Chaidos A, Olavarria E, Goldman JM, Apperley JF, et al: Pleural effusions in patients with chronic myeloid leukaemia treated with dasatinib may have an immunemediated pathogenesis. Br J Haematol 141: 745-747, 2008.

19. Mustjoki S, Ekblom M, Arstila TP, Dybedal I, EplingBurnette PK, Guilhot F, Hjorth-Hansen H, Höglund M, Kovanen P, Laurinolli T, et al: Clonal expansion of T/NK-cells during tyrosine kinase inhibitor dasatinib therapy. Leukemia 23: 1398-1405, 2009 
20. Kreutzman A, Juvonen V, Kairisto V, Ekblom M, Stenke L, Seggewiss R, Porkka K and Mustjoki S: Mono/oligoclonal T and NK cells are common in chronic myeloid leukemia patients at diagnosis and expand during dasatinib therapy. Blood 116: 772-782, 2010.

21. Kim DH, Kamel-Reid S, Chang H, Sutherland R, Jung CW, Kim HJ, Lee JJ and Lipton JH: Natural killer or natural killer/T cell lineage large granular lymphocytosis associated with dasatinib therapy for Philadelphia chromosome positive leukemia. Haematologica 94: 135-139, 2009.
22. Chuah CT, Nakamae H, Shen ZX, Bradley-Garelik MB and Kim DW: Efficacy and safety of dasatinib versus imatinib in the East Asian subpopulation of the DASISION trial of newly diagnosed chronic myeloid leukemia in chronic phase. Leuk Lymphoma 55: 2093-2100, 2014.

23. Fujisawa S, Nakamae H, Ogura M, Ishizawa K, Taniwaki M, Utsunomiya A, Matsue K, Takamatsu Y, Usuki K, Tanimoto M, et al: Efficacy and safety of dasatinib versus imatinib in Japanese patients with newly diagnosed chronic-phase chronic myeloid leukemia (CML-CP): Subset analysis of the DASISION trial with 2-year follow-up. Int J Hematol 99: 141-153, 2014. 